\title{
Oncogene ATAD2 promotes cell proliferation, invasion and migration in cervical cancer
}

\author{
LE ZHENG，TIANREN LI，YI ZHANG，YI GUO，JIHANG YAO, \\ LEI DOU and KEJUN GUO
}

\begin{abstract}
Department of Gynecology, The First Hospital of China Medical University, Shenyang, Liaoning 110001, P.R. China
\end{abstract}

Received December 12, 2014; Accepted March 8, 2015

DOI: $10.3892 /$ or.2015.3867

\begin{abstract}
The ATPase family AAA domain-containing protein 2 (ATAD2) is associated with many cellular processes, such as cell proliferation, invasion and migration. However, the molecular biological function of the ATAD2 gene in cervical cancer is unclear. The purpose of this study was to explore ATAD2 expression in cervical cancer, evaluate the relationship between the development of cervical cancer, metastasis and clinicopathological characteristics, and discuss the implications for its use in clinical treatment. Protein and mRNA expression of ATAD2 was examined in tissues and cell lines. Tumor tissues from 135 cases of cervical cancer were collected for evaluation of ATAD2 expression by immunohistochemistry and western blotting. Prognostic significance was evaluated by the Cox hazards model and Kaplan-Meier survival method. HeLa and SiHa cells were transfected with two siRNAs targeting ATAD2. ATAD2 knockdown was used to analyze cell proliferation, invasion and migration. Cell viability was evaluated with the Cell Counting Kit-8 (CCK-8) assay, cell invasion by a Transwell assay and cell migration by a wound healing/scratch migration assay. ATAD2 was shown to be highly expressed in cervical cancer tissues, both at the transcriptional and protein levels, and was correlated with poor patient survival $(\mathrm{P}<0.05)$. Knockdown of ATAD2 in the HeLa and $\mathrm{SiHa}$ cells was found to reduce the capacity for invasion and migration $(\mathrm{P}<0.05)$, and inhibited the growth and clonogenic potential of the HeLa and SiHa cell lines. Our results suggest that cervical cancer tissues may have highly expressed ATAD2, which is associated with tumor stage and lymph node status $(\mathrm{P}<0.05)$. Oncogene ATAD2 may play an important role in cervical cancer proliferation, invasion and migration. It could serve as a prognostic marker and a therapeutic target for cervical cancer.
\end{abstract}

Correspondence to: Dr Kejun Guo, Department of Gynecology, The First Hospital of China Medical University, Shenyang, Liaoning 110001, P.R. China

E-mail: guokejuncmu@126.com

Key words: ATPase family AAA domain-containing protein 2, cervical cancer, prognostic marker, proliferation

\section{Introduction}

Cervical cancer is the second most common malignant tumor in females worldwide. It has been estimated to affect 500,000 women/year and lead to 270,000 deaths (1). In recent years, there has been considerable progress in the diagnosis and comprehensive treatment of cervical cancer. Nevertheless, there have been no fundamental changes in cervical cancer incidence and mortality. Cervical cancer occurrence and development is a gradual process. Cervical intraepithelial neoplasia (CIN) is one of the common precancerous lesions of cervical cancer. High-risk human papilloma virus (HPV) infection is closely related to cervical precancerous lesions and cervical cancer. However, a single HPV infection will not necessarily develop into cervical cancer (2). Cervical cancer is also related to the genetic and immune system of the host and other factors. It is believed that the occurrence and development of cervical cancer is a complex multi-factor and multi-step process, often involving proto-oncogenes and activation or inhibition of tumor-suppressor genes (3). Therefore, there is a great demand to identify reliable prognostic biomarkers, to predict the tendency of cervical cancer metastasis, to detect recurrent lesions early, and to standardize treatment, all of which may improve the overall survival rate and reduce mortality.

ATPase family AAA domain-containing protein 2 (ATAD2), a member of the AAA+ ATPase family, also known as ANCCA (AAA+ nuclear coregulator cancer-associated), was identified by microarray analysis (4). ATAD2 is a remarkably conserved protein that is expressed predominantly in germ cells, but is systematically overexpressed in many different types of unrelated cancers (5). ATAD2 contains both an ATPase domain and bromodomain. The presence of a bromodomain adjacent to an AAA type ATPase domain indicates that ATAD2 is a factor acting primarily on chromatin structure and function (6). The structure of ATAD2 suggests that it has functions associated with genome regulation, including cell proliferation, differentiation and apoptosis (7). ATAD2 can regulate downstream genes such as cyclin D1, c-Myc and E2F1, which contribute directly or indirectly to cell proliferation (8). Studies have revealed that ATAD2 is highly expressed in several types of tumors, such as breast, ovarian, endometrial and lung cancer, hepatocellular carcinoma and 
large B-cell lymphoma (9-14). However, there have been no studies concerning the gene function and prognosis related to ATAD2 in cervical cancer.

In the present study, we compared ATAD2 levels in cervical cancer specimens and matched normal/tumoradjacent cervical tissues, in order to investigate the impact of ATAD2 on cell proliferation, invasion and migration. Different methods were used to determine the relationships between the ATAD2 level and clinicopathological characteristics and to elucidate its prognostic value in cervical cancer patients based on survival data.

\section{Materials and methods}

Patient tissue samples. One hundred and thirty-five cervical cancer (CC) and sixty CIN tissues were collected from patients who underwent routine cervical resection at the First Affiliated Hospital of China Medical University. None of the patients had received preoperative radiotherapy or chemotherapy prior to surgical resection. Clinical stage and grade were evaluated independently by two pathologists according to the WHO classification system. Clinical characteristics of all patients, including the histological type, FIGO stage, histological differentiation grade and lymph node statues are described in Table I. Total RNAs and protein were collected from the fresh CC and CIN tissues after surgical resection. The project protocol was approved by the Institutional Ethics Committee of China Medical University prior to the initiation of the study, and all patients provided written informed consent.

Immunohistochemistry. ATAD2 expression was analyzed on 4- $\mu \mathrm{m}$ paraffin-embedded specimens. Rabbit anti-ATAD2 (1:200, Sigma-Aldrich, St. Louis, MO, USA) was used. Sections were stained with 3,3'-diaminobenzidine (DAB). ATAD2 expression levels were scored semi-quantitatively according to the percent of positively stained cells combined with the staining intensity. Samples were considered positive for ATAD2 if the nucleus/cytoplasm of the sample cells presented positive staining. The percent positivity was defined as follows: 0 (0\%), 1 (1-10\%), 2 (11-50\%), 3 (51-80\%), or $4(>80 \%)$. The staining intensity was scored as follows: 0 (no staining), 1 (weakly stained), 2 (moderately stained) and 3 (strongly stained). Both the staining intensity and the percent positivity were assessed by two investigators in a doubleblinded manner. The ATAD2 expression score was calculated from the product of the percent positivity score $\mathrm{x}$ the staining intensity score. Each sample was given a final score ranging from 0 to 12 , and the tumors were classified as follows: negative (-), score 0 ; low expression (1+), score 1-4; moderate expression (2+), score 5-8; and strong expression $(3+)$, score 9-12. The immunohistochemical ATAD2 staining was grouped into two categories: low expression (0 to 1+) and high expression $(2+$ to $3+)$.

Western blot analysis. Cells or tissues were harvested, washed in PBS and placed in an appropriate volume of lysis buffer (Beyotime Biotechnology, Beijing, China). Total protein lysates $(50 \mu \mathrm{g})$ were boiled in SDS-PAGE sample buffer for $10 \mathrm{~min}$, then loaded onto $12 \%$ SDS-PAGE gels and transferred
Table I. Correlation of ATAD2 expression and the clinicopathological characteristics of the 135 cervical cancer patients.

\begin{tabular}{llll}
\hline & \multicolumn{2}{c}{ ATAD2 expression } & \\
\cline { 2 - 3 } Characteristics & High, n (\%) & Low, n (\%) & P-value \\
\hline Total cases & $96(71.1)$ & $39(28.9)$ & \\
Age (years) & & & 0.493 \\
$\geq 45$ & $53(73.6)$ & $19(26.4)$ & \\
<45 & $43(68.3)$ & $20(31.7)$ & \\
Histological type & & & 0.578 \\
Squamous & $72(69.9)$ & $31(30.1)$ & \\
Adenocarcinoma & $24(80.0)$ & $8(20.0)$ & \\
FIGO stage & & & \\
I-IIa & $41(62.1)$ & $25(37.9)$ & \\
IIb-IV & $55(79.7)$ & $14(20.3)$ & \\
Differentiation & & & \\
Well & $31(62.0)$ & $19(38.0)$ & \\
Moderate & $27(73.0)$ & $10(27.0)$ & \\
Poor & $38(79.2)$ & $10(20.8)$ & \\
Lymph node status & & & \\
Positive & $46(80.7)$ & $11(19.3)$ & \\
Negative & $50(64.1)$ & $28(35.9)$ & \\
\hline
\end{tabular}

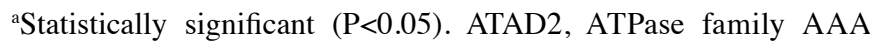
domain-containing protein 2 .

to PVDF membranes (Millipore, Billerica, MA, USA) after electrophoresis. After blocking, the primary ATAD2 and GAPDH mouse polyclonal antibodies (both diluted at 1:1,000; Sigma-Aldrich) were incubated with the membranes at $4^{\circ} \mathrm{C}$ overnight. The secondary antibodies were incubated for $2 \mathrm{~h}$ at room temperature. Protein bands were visualized using an ECL system (Millipore, Bedford, MA, USA) .

Cell culture and RNA interference. The CC cell lines, HeLa and SiHa were obtained from the Chinese Academy of Sciences Cell Bank (Shanghai, China) and cultured in Dulbecco's modified Eagle's medium (DMEM)/RPMI1640 (Invitrogen, Carlsbad, CA, USA). All media were supplemented with $10 \%$ fetal calf serum. ATAD2 siRNAs (purchased from GenePharma Co., Ltd., Shanghai, China) were: GGAUCUCUCUUCAAUUAAUT (si-ATAD2\#1) and GUGCGUCGAAGUUGUAGGATT (si-ATAD2\#2). For transfections, $24 \mathrm{~h}$ after the cells were seeded in a 24 -well plate, they were transfected with the ATAD2 siRNAs or the negative control siRNA (Neg. siRNA) using Dharma FECT 1 (Thermo Fisher Scientific, Waltham, MA, USA) according to the manufacturer's instructions. The mRNA and protein levels were detected $48 \mathrm{~h}$ later.

Real-time quantitative PCR ( $q P C R)$. Total RNA was extracted from the HeLa and SiHa cells which were transfected with ATAD2 siRNAs or Neg. siRNA with TRIzol reagent (Takara Biotechnology, Dalian, China). Real-time quantitative 

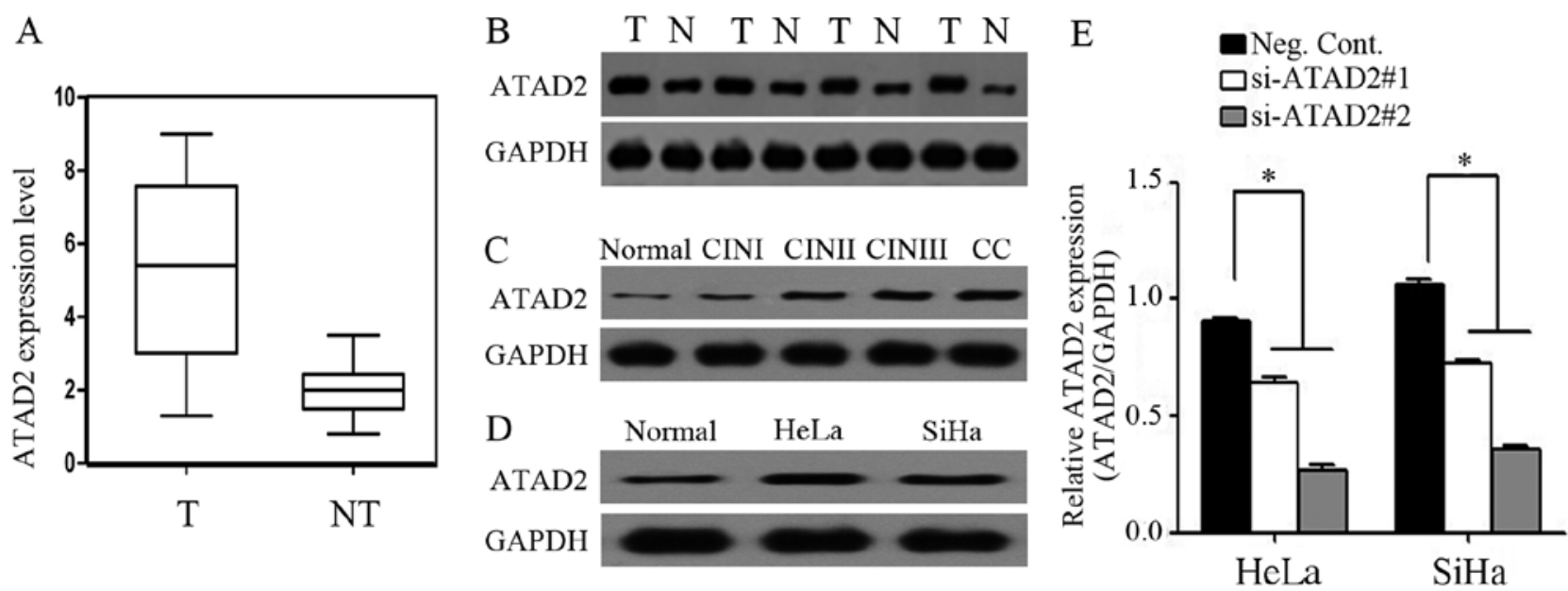

Figure 1. ATAD2 expression level in the CC tissues and CC cell lines. (A) Mean level of ATAD2 expression was determined by western blotting in 30 pairs of tumor (T) and non-tumor (NT) tissues. (B) ATAD2 protein expression in each of the tumor (T) and paired non-tumor (NT) samples was determined by western blotting. (C) The expression of ATAD2 protein identified by western blotting in CC, CIN and normal tissues. (D) The expression of ATAD2 in HeLa and SiHa cells compared with normal cervical epithelial samples (Normal). (E) The efficiency of RNA interference by two short RNAs (si-ATAD2\#1 and si-ATAD2\#2) in the HeLa and SiHa cells was detected by qPCR. CC, cervical cancer. CIN, cervical intraepithelial neoplasia; ATAD2, ATPase family AAA domain-containing protein 2.

PCR (qPCR) was performed using a SYBR Premix ExTaq on a Thermal Cycler Dice Real-Time system (both from Takara Biotechnology) with the following parameters: $30 \mathrm{sec}$ at $95^{\circ} \mathrm{C}$ followed by a two-step PCR for 40 cycles of $95^{\circ} \mathrm{C}$ for $5 \mathrm{sec}$ and $64^{\circ} \mathrm{C}$ for $30 \mathrm{sec}$. Each reaction was performed as previously described. The primer sequences were: ATAD2 forward, 5'-GGAATCCCAAACCACTGGACA-3' and reverse, 5'-GGT AGCGTCGTCGTAAAGCACA-3'; GAPDH forward, 5'-ATAGCACAGCCTGGATAGCAACGTAC-3' and reverse, 5'-CACCTTCTACAATGAGCTGCGTGTG-3'. The experiments were repeated in triplicate. The relative levels of gene expression were represented as $\Delta \mathrm{Ct}=\mathrm{Ct}$ gene $-\mathrm{Ct}$ reference, and the fold change of gene expression was analyzed by the $2^{-\Delta \Delta \mathrm{Ct}}$ method.

Cell Counting Kit-8 (CCK-8) and colony formation assay. For the CCK-8 assay, cells were plated in 96-well plates in media containing 10\% FBS at 2,000 cells/well, $24 \mathrm{~h}$ after transfection. Then, $10 \mu \mathrm{l}$ of CCK-8 (KeyGen Bio., Nanjing, China) solution was added to each well and incubated for $1 \mathrm{~h}$ at $37^{\circ} \mathrm{C}$. The results were quantitated using a test wavelength of $450 \mathrm{~nm}$. For the colony formation assay, the colony formation was allowed to proceed for 14 days. Plates were stained with $0.1 \%$ crystal violet. The fixed cell colonies were allowed to air dry. The clone formation rate was calculated.

Cell cycle analysis. HeLa and SiHa cells in 6-well plates were transfected with ATAD2 siRNA (si-ATAD2\#2) or Neg. siRNA. After $24 \mathrm{~h}$ of transfection, the cells were seeded at a density of $5 \times 10^{5}$ cells/well, trypsinized, fixed with $70 \%$ cold ethanol at $4^{\circ} \mathrm{C}$, and washed in cold PBS. A quantity of $100 \mu 1$ RNase A and $400 \mu \mathrm{l}$ PI staining solution was added, and the cells were incubated at $4^{\circ} \mathrm{C}$ in the dark for $30 \mathrm{~min}$. Cell cycle analysis was performed using a flow cytometer (FACSCalibur; BD Biosciences, San Jose, CA, USA). A software was then used to detect and record the red fluorescence upon excitation at a wavelength of $488 \mathrm{~nm}$.
Wound healing/scratch migration and Transwell assays. For the wound healing/scratch migration assay, the cells were seeded into 6-well plates at a density that after $24 \mathrm{~h}$ of growth, they reached $\sim 60 \%$ confluency as a monolayer. The monolayer was gently and slowly scratched with a new pipette tip across the center of the well. After scratching, the well was washed twice with PBS to remove the detached cells and was then replenished with fresh medium. The cells were grown for an additional $24 \mathrm{~h}$. Images were captured of the monolayer using a microscope. The gap distance was quantitatively evaluated using software. For the Transwell assay, the HeLa and SiHa cells, with $200 \mu \mathrm{l}$ serum-free media were added to the upper compartment which was coated with $30 \mu 1$ Matrigel (1:2 dilution; Costar, Corning, NY, USA). RMPI-1640 containing $10 \%$ FBS was added to the lower compartment, and further incubation was carried out for $24 \mathrm{~h}$. The cells that penetrated the membrane of the chamber were stained with hematoxylin. The cells on the upper membrane were removed with a cotton tip. The number of invasive cells was assessed in 10 randomly selected fields under a microscope. The experiments were tested in triplicate.

Statistical analysis. The statistical data were analyzed by SPSS 17.0 for Windows. The correlation between ATAD2 expression and cervical cancer patient clinicopathological features was analyzed using the $\chi^{2}$ test. Student's t-test analysis was used to compare messenger RNA in the various groups. Prognostic significance was evaluated by the Cox hazards model and Kaplan-Meier survival method. A difference was considered statistically significant at $\mathrm{P}<0.05$.

\section{Results}

Expression of ATAD2 in the CC tissue samples and cell lines. At the protein level, the mean level of ATAD2 expression in the CC tissues was 3.24-fold higher than that in the non-tumor tissues (median expression, 5.157 and 1.917, respec- 

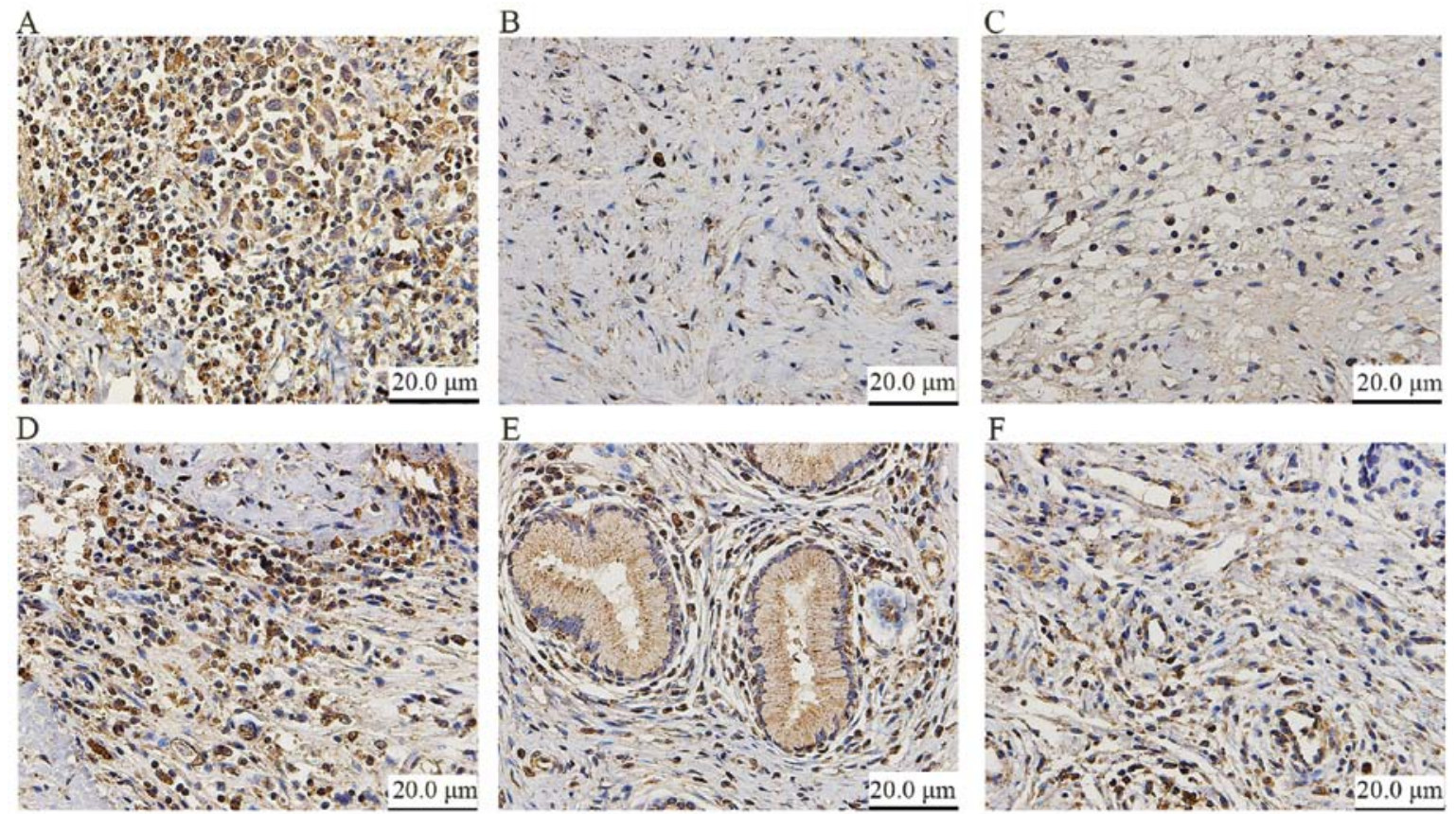

Figure 2. Immunohistochemical staining of ATAD2 in the CC and non-tumor samples. High expression in the (A) CC and (D) CINIII tissues. Low expression in the (E) CIN II and (F) CINI tissues. Negative expression in the (B) tumor-adjacent and (C) normal cervix tissues (immunohistochemistry, $\mathrm{x} 400$ ). CC, cervical cancer; CIN, cervical intraepithelial neoplasia; ATAD2, ATPase family AAA domain-containing protein 2.

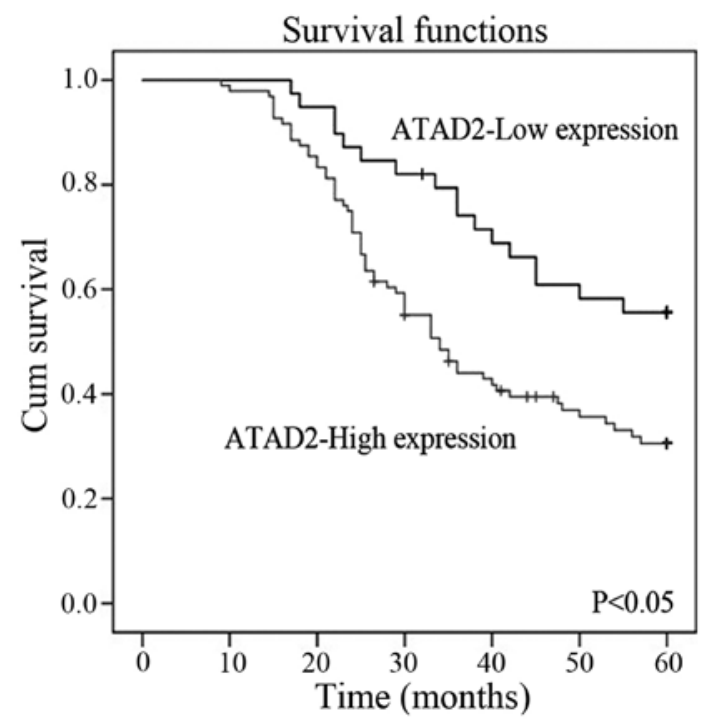

Figure 3. Survival time of the cervical cancer patients in relation to ATAD2 expression. Overall survival of the cervical cancer patients with high and low ATAD2 expression is shown. ATAD2, ATPase family AAA domain-containing protein 2 .

tively) (Fig. 1A). Western blot analysis showed that ATAD2 expression was markedly higher in the $\mathrm{CC}$ tissues compared to their adjacent normal cervical tissues (Fig. 1B), and ATAD2 expression in the CC and CINII-III tissues was higher than that in the normal cervical and CINI tissues (Fig. 1C). Compared to the normal cell line, HeLa and SiHa cells showed relatively higher levels of ATAD2 expression by western blotting (Fig. 1D). In addition, ATAD2 expression in the HeLa and $\mathrm{SiHa}$ cells transfected with the ATAD2 siRNA showed efficient depletion (Fig. 1E). According to the immunohisto-
Table II. Expression of ATAD2 in CC, CIN and normal cervical tissues.

\begin{tabular}{lrcc}
\hline Tissues & $\mathrm{n}$ & High, $\mathrm{n}(\%)$ & P-value \\
\hline Normal cervical & 30 & $6(20.0)$ & \\
CINI & 30 & $8(26.7)$ & 0.542 \\
CINII-III & 30 & $16(53.3)$ & $0.007^{\mathrm{a}}$ \\
CC & 135 & $96(71.1)$ & $<0.001^{\mathrm{a}}$ \\
\hline
\end{tabular}

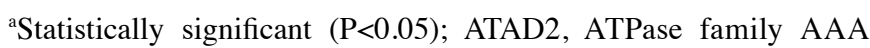
domain-containing protein 2 ; CC, cervical cancer; CIN, cervical intraepithelial neoplasia.

chemical staining, ATAD2 was highly expressed in the CC tissues $(96 / 135,71.1 \%)$ and CINIII tissues $(16 / 30,53.3 \%)$, whereas little or no staining was observed in the tumoradjacent and normal cervical tissues (Fig. 2). Expression of ATAD2 in the CC, CINII-III, CINI, and normal cervical tissues is summarized in Table II.

Correlation of ATAD2 expression with cervical cancer patient clinical outcome. High levels of ATAD2 expression were significantly associated with FIGO stage and lymph node status. Association of ATAD2 expression and the clinicopathological characteristics of the 135 cervical cancer patients are summarized in Table I. Overall survival was significantly reduced in patients with high ATAD2 expression compared to patients with low ATAD2 expression ( $\mathrm{P}<0.05$, Fig. 3). In addition, a multivariate analysis demonstrated that ATAD2 status, FIGO stage and lymph node status were significant prognostic factors for cervical cancer patients. A high level of ATAD2 expression was 
Table III. Univariate and multivariate analyses of the individual parameters correlated with overall survival of the cervical cancer patients.

\begin{tabular}{|c|c|c|c|c|c|c|}
\hline \multirow[b]{2}{*}{ Variables } & \multicolumn{3}{|c|}{ Univariate analysis } & \multicolumn{3}{|c|}{ Multivariate analysis } \\
\hline & HR & $95 \% \mathrm{CI}$ & P-value & HR & $95 \% \mathrm{CI}$ & P-value \\
\hline ATAD2 & 2.509 & $1.530-4.115$ & $<0.001^{\mathrm{a}}$ & 1.803 & $1.180-2.756$ & $0.006^{\mathrm{a}}$ \\
\hline Age & 1.019 & 0.993-1.046 & 0.156 & & & \\
\hline FIGO stage & 3.651 & $2.360-5.648$ & $<0.001^{\mathrm{a}}$ & 1.852 & $1.214-2.285$ & $0.004^{\mathrm{a}}$ \\
\hline Differentiation & 1.118 & $0.881-1.419$ & 0.357 & & & \\
\hline Histological type & 1.194 & $0.680-1.759$ & 0.711 & & & \\
\hline Lymph node status & 3.985 & $2.256-5.309$ & $<0.001^{\mathrm{a}}$ & 2.533 & $1.665-3.852$ & $0.001^{\mathrm{a}}$ \\
\hline
\end{tabular}

${ }^{a}$ Statistically significant $(\mathrm{P}<0.05)$. HR, hazard ratio; CI, confidence interval.
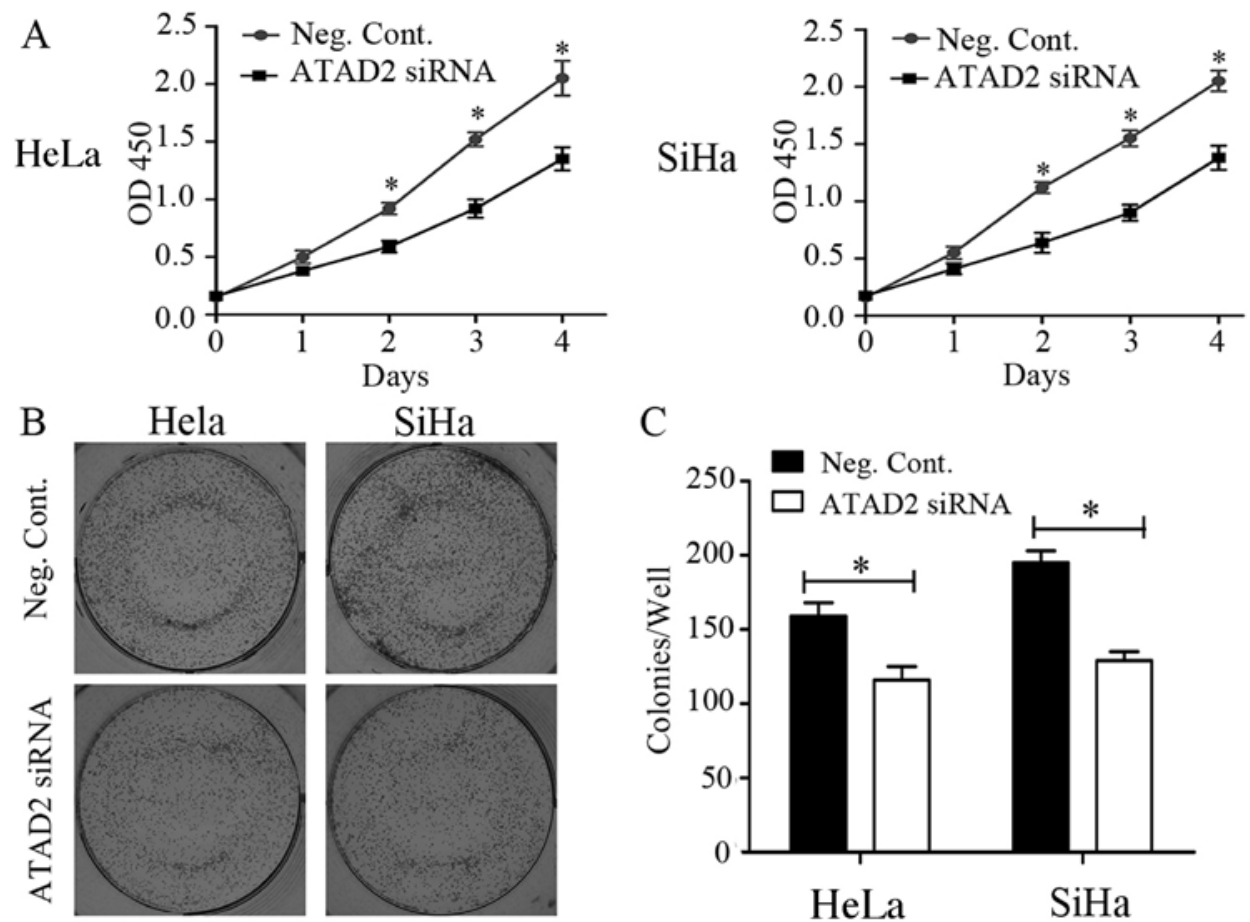

$\mathrm{SiHa}$

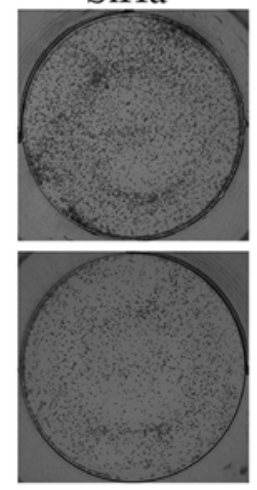

C

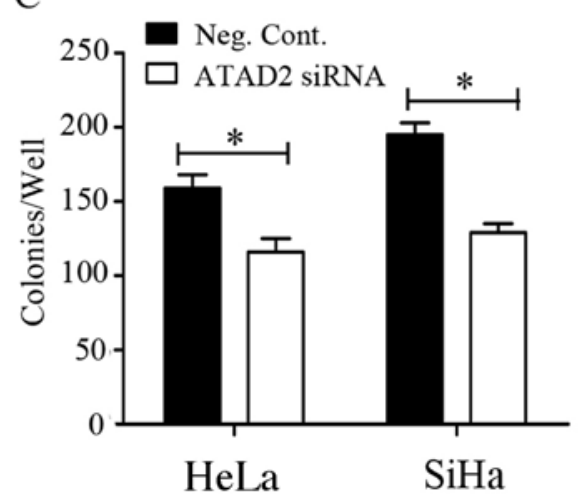

Figure 4. ATAD2 depletion impairs cell proliferation. (A) The CCK-8 assay was performed following ATAD2 siRNA treatment. ATAD2 was knocked down by si-ATAD2\#2, which inhibited HeLa and SiHa cell growth. A reduction in absorbance was observed ( $\mathrm{P}<0.05)$. (B) Clonogenic assays were performed with ATAD2-depleted cancer cells. (C) The number of colonies formed by the HeLa and SiHa cells treated with ATAD2 siRNA (si-ATAD2\#2) was markedly less than the number of colonies in the control siRNA-treated cells ( $\mathrm{P}<0.05)$. Each of the experiments was repeated in triplicate.

associated with increased risk of a poor prognosis (hazard ratio, 1.803, $\mathrm{P}=0.006$ ) (Table III).

Depletion of ATAD2 inhibits tumor cell proliferation in the $C C$ cell lines. A significant reduction in the proliferation rate of the $\mathrm{CC}$ cell lines was observed with the CCK-8 assay after transfection with ATAD2 siRNA compared to Neg. siRNA (Fig. 4A). Consistent with the CCK-8 assay, the depletion of ATAD2 in the HeLa (Neg. siRNA vs. ATAD2 siRNA: $159 \pm 9$ vs. $116 \pm 9, \mathrm{P}<0.05)$ and $\mathrm{SiHa}$ (Neg. siRNA vs. ATAD2 siRNA: $195 \pm 8$ vs. $129 \pm 6, \mathrm{P}<0.05$ ) cells led to a significant reduction in the number and size of foci (Fig. 4B). The DNA content determined using flow cytometry revealed that ATAD2 siRNA transfection increased the percentage of cells in the G1 phase and decreased those in the $\mathrm{S}$ phase in both the HeLa and $\mathrm{SiHa}$ cell lines $(\mathrm{P}<0.05$, Fig. 5).

Invasive and migratory capacities of the HeLa and SiHa cells are decreased by ATAD2 knockdown. Cell invasion and migration assays demonstrated that the HeLa and SiHa CC cell lines that were transfected with ATAD2 siRNA displayed attenuated invasive and migratory capacities than these capacities in the negative controls (Figs. 6 and 7). The depletion of ATAD2 in the HeLa (Neg. siRNA vs.ATAD2 siRNA: $263.7 \pm 9.5$ vs. $142.7 \pm 5.0, \mathrm{P}<0.05)$ and $\mathrm{SiHa}(\mathrm{Neg}$. siRNA vs. ATAD2 siRNA: 

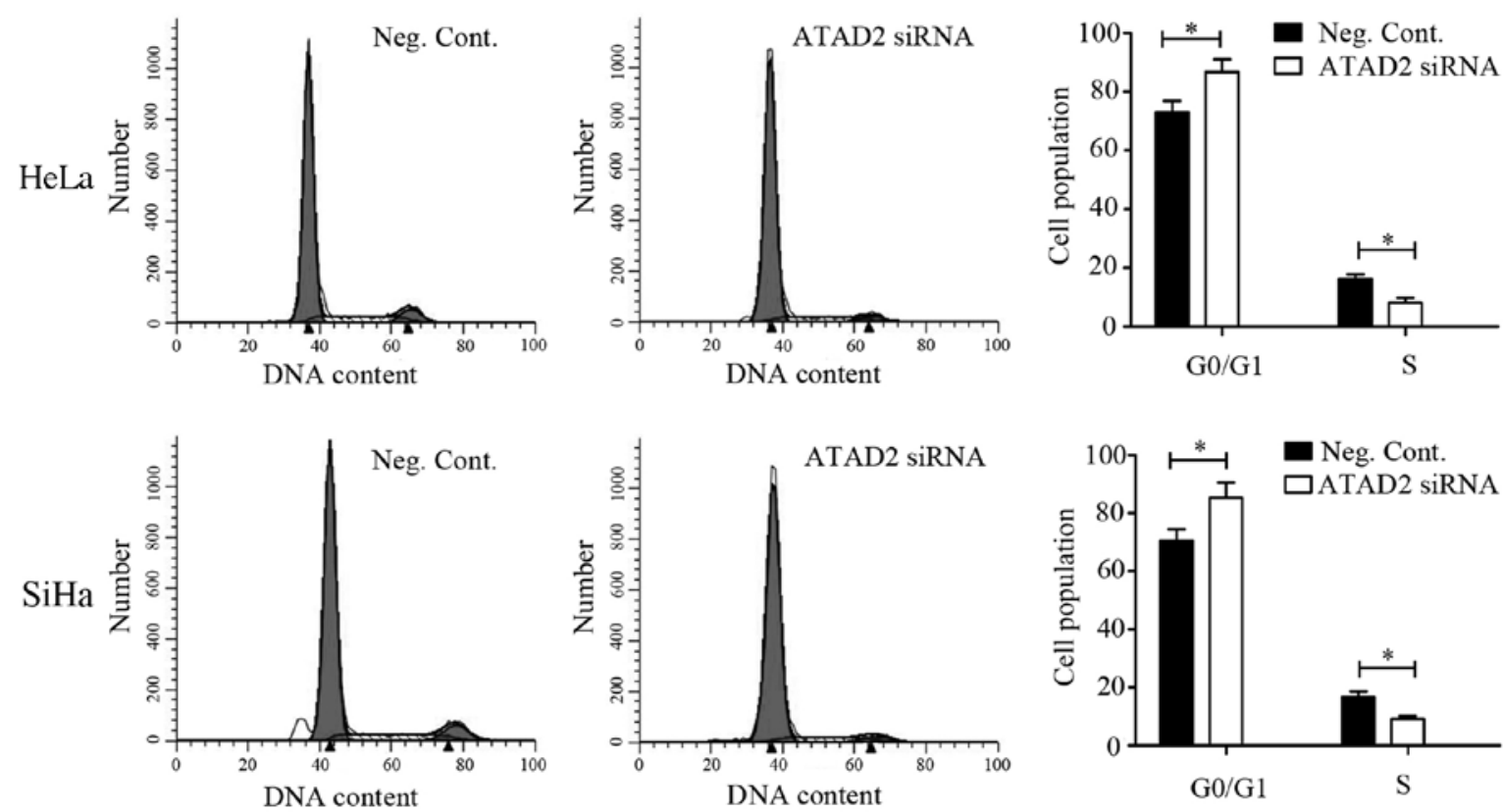

Figure 5. DNA content analysis demonstrated that ATAD2 siRNA (si-ATAD2\#2) transfection increased the percentage of cells in the G1 phase and decreased those in the S-phase in the HeLa and SiHa cell lines. ATAD2, ATPase family AAA domain-containing protein 2.
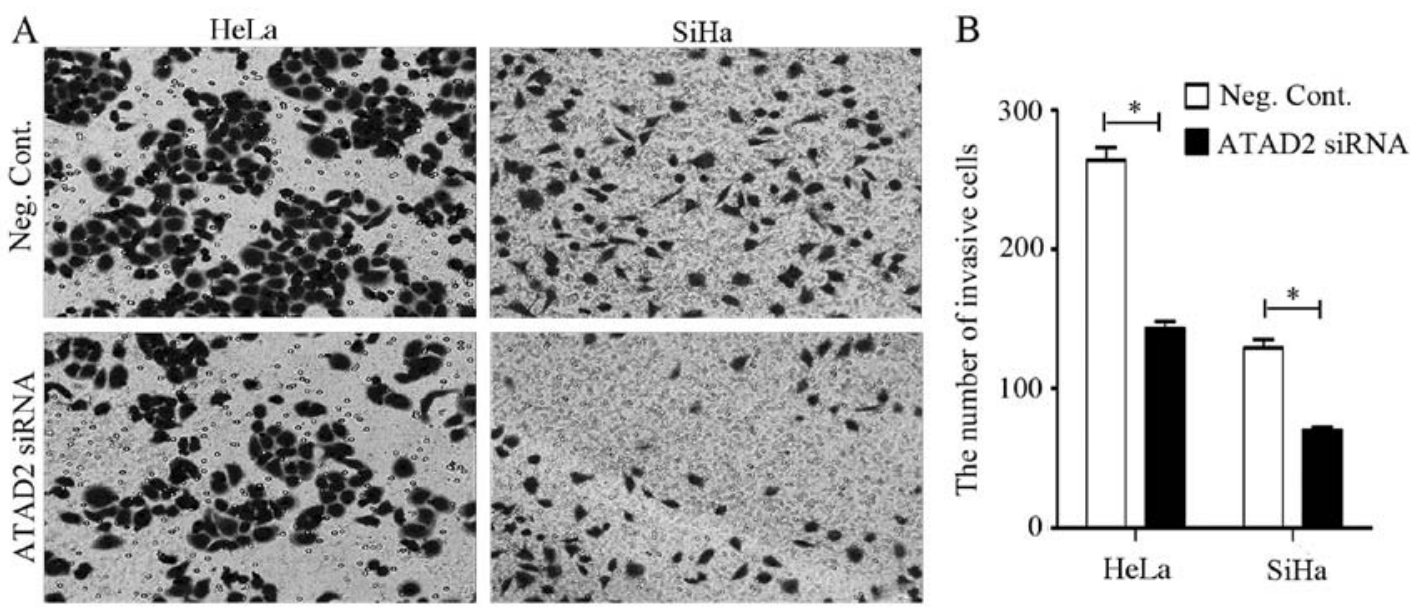

Figure 6. Transwell assays of the HeLa and SiHa cells transfected with the negative control and ATAD2 siRNA (si-ATAD2\#2). (A) ATAD2 depletion had a measurable inhibitory effect on cell invasion in both cell lines. (B) The number of invasive cells was counted and a significant difference was observed among the cell groups ( $\left.{ }^{*} \mathrm{P}<0.05\right)$. ATAD2, ATPase family AAA domain-containing protein 2.

$128.7 \pm 5.5$ vs. $70.0 \pm 2.0, \mathrm{P}<0.05)$ cells led to a significant reduction in invasive cells (Fig. 6). The migratory ability of the HeLa and $\mathrm{SiHa}$ cells was assessed by a wound healing/scratch migration assay. Migration of the HeLa (Neg. siRNA vs. ATAD2 siRNA: $164.57 \pm 2.31$ vs. $234.31 \pm 1.65, \mathrm{P}<0.05)$ and $\mathrm{SiHa}$ (Neg. siRNA vs. ATAD2 siRNA: $181.87 \pm 8.08$ vs. $255.05 \pm 3.53$, $\mathrm{P}<0.05$ ) cells was also significantly reduced (Fig. 7 ).

\section{Discussion}

Discovery of the ATAD2 gene is a new breakthrough in oncology research. The AAA+ ATPase structure domain is a molecular chaperone using ATP activity to participate in the activities of cells, cell cycle control, signal transduction, and gene expression regulation (15). The bromine structure domain and acetylation of the lysine model adjust chromosome remodeling and transcriptional control of the interaction between proteins (16-19). ATAD2 maps to chromosome $8 \mathrm{q} 24$ in a region that is frequently amplified in cancer (20). It was demonstrated that ATAD2 is a new type of candidate oncogene and perhaps a therapeutic target for several types of human tumors, such as breast, ovarian, endometrial and lung cancer and hepatocellular carcinoma (9-13). However, the abnormal expression of ATAD2 and its possible role in the carcinogenesis of cervical cancer have not been studied to date.

In the present study, immunohistochemical analysis showed that the expression of ATAD2 in cervical cancer tissues was significantly higher than that in the normal/tumor-adjacent tissues. ATAD2 expression in the CINII-III tissues was 

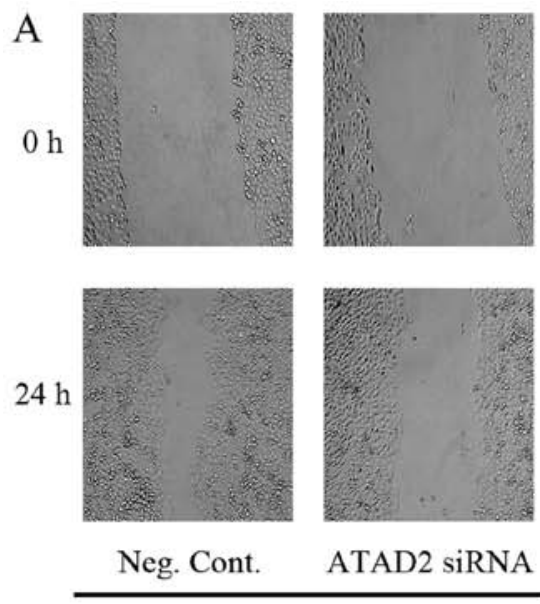

HeLa
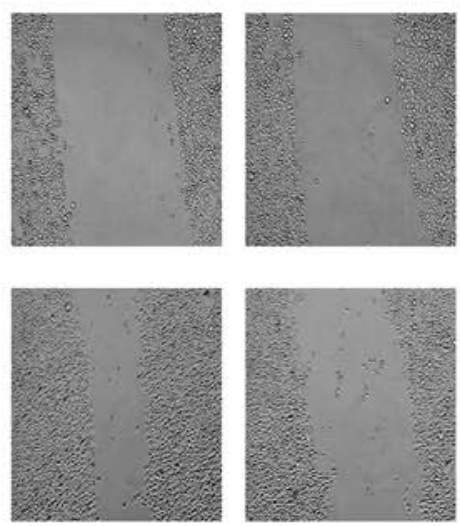

Neg. Cont.
B
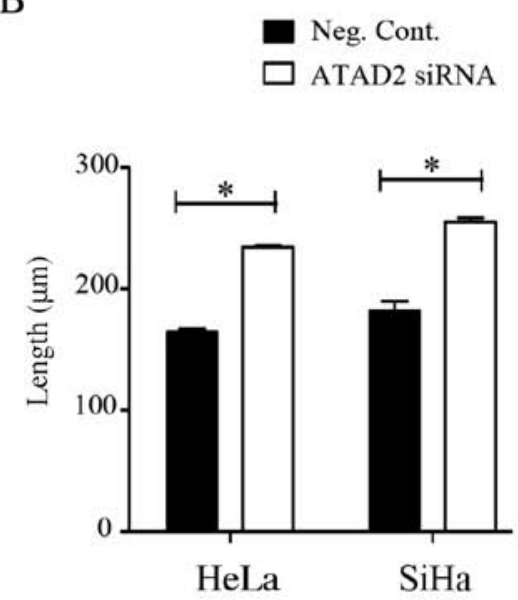

Figure 7. Wound healing/scratch migration assays of the HeLa and SiHa cells transfected with the negative control and ATAD2 siRNA (si-ATAD2\#2). (A) ATAD2 depletion had a measurable inhibitory effect on cell migration in both cell lines. (B) The number of migratory cells was counted and a significant difference was observed ( $\mathrm{P}<0.05)$. Results are representative of three independent experiments. ATAD2, ATPase family AAA domain-containing protein 2.

significantly higher than that in the CINI and normal tissues. The same results were demonstrated by western blotting. A total of 135 cervical cancer tissue samples were analyzed by $\chi^{2}$ test. The results indicated that ATAD2 is one type of cervical cancer gene and is related to cervical cancer proliferation and transference. We found that high expression of ATAD2 in cervical cancer patients was an independent predictor of overall survival. Overexpression of the ATAD2 gene may play an important role in the occurrence and development of cervical cancer, but the specific mechanism and genes for cervical cancer cell proliferation warrant further research.

Previous studies showed that ATAD2 was highly expressed in a variety of human tumor cell lines, including those derived from leukemia (HL60, Molt4 and Jurkat), lymphoma (Daudi), lung adenocarcinoma (A549 and A1299), and breast cancer (MCF-7) (21). ATAD2 in human cancer cells is associated with a variety of key regulatory mechanisms, such as regulation of cell proliferation and tumor metastasis $(22,23)$. ATAD2 can regulate downstream genes such as cyclin D1, c-myc and E2F, which contribute indirectly or directly to cancer cell proliferation (24). High expression of ATAD2 can promote cell proliferation in breast cancer and is closely related to tumor stage (9). Through small RNA interference, tumor cell proliferation ability was decreased, and apoptosis was induced in breast cancer cells (21). In prostate cancer, ATAD2 also plays an important role in tumor cell proliferation (25). At present, how ATAD2 affects cell proliferation and tumor molecular mechanisms is unclear.

A recent study showed that ATAD2 and some important mitotic drive protein subtype genes and cell survival genes (IRS2, VEGF and AKtl) controlled by c-myc and EZH2 are associated with proliferation of Rb-E2F (26). ATAD2 exists in the E2F sensitive gene promoter, and when the cells change from the G1 to the S phase, ATAD2 was increased significantly (27). In our research, the HeLa and SiHa cells had relatively higher expression of ATAD2, and the depletion of ATAD2 by transfection with siRNA in these two cell lines led to G1 phase cell cycle arrest and reduced proliferation and colony-forming ability. This result was in agreement with previous studies, and siRNA-mediated ATAD2 knockdown significantly inhibited cervical cancer cell invasion and migration. In agreement with the literature, through activation of inflammatory factors such as nuclear factor $-\kappa \mathrm{B}(\mathrm{NF}-\kappa \mathrm{B})$, ATAD2 plays a main role in tumor progression. Histone methyltransferase NSD2 (also known as MMSET and WHSC1), an NF- $\kappa \mathrm{B}$ activator, is a construction of bromine ANCCA/ ATAD2 gene of target genes. It activates downstream target genes, including IL-6, IL-8, cyclin D and Bcl-2. A previous study suggests that the downstream target genes may play an important role in cervical cancer cell metastasis (28). Our results provide evidence that ATAD2 is not only important in cervical cancer cell proliferation, but is also involved in cervical cancer cell invasion and migration. Further research is necessary to understand the specific mechanisms.

In summary, the present study identified high expression of the oncogene ATAD2 in cervical cancer, which may be important in the acquisition of an aggressive phenotype and may indicate a poor prognosis for cervical cancer patients. In addition, the functional studies of ATAD2 suggest that it has a potential role in regulating cell proliferation, invasion, and migration. ATAD2 is a candidate target protein for future cancer therapeutics.

\section{Acknowledgements}

This study was supported by grants from the National Natural Science Foundation of China (no. 81171649 awarded to Y.G.).

\section{References}

1. Khenchouche A, Sadouki N, Boudriche A, Houali K, Graba A, Ooka $\mathrm{T}$ and Bouguermouh A: Human papillomavirus and Epstein-Barr virus co-infection in cervical carcinoma in Algerian women. Virol J 10: 340, 2013.

2. Thomison J III, Thomas LK and Shroyer KR: Human papillomavirus: molecular and cytologic/histologic aspects related to cervical intraepithelial neoplasia and carcinoma. Hum Pathol 39: 154-166, 2008.

3. Xiao S, Liao S, Zhou Y, Jiang B, Li Y and Xue M: High expression of octamer transcription factor 1 in cervical cancer. Oncol Lett 7: 1889-1894, 2014 
4. Zou JX, Revenko AS, Li LB, Gemo AT and Chen HW: ANCCA, an estrogen-regulated AAA+ ATPase coactivator for ERalpha, is required for coregulator occupancy and chromatin modification. Proc Natl Acad Sci USA 104: 18067-18072, 2007.

5. Boussouar F, Jamshidikia M and Morozumi Y: Malignant genome reprogramming by ATAD2. Biochim Biophys Acta 10: 1010-1014, 2013.

6. Ciró M, Prosperini E, Quarto M, Grazini U, Walfridsson J, McBlane F, Nucifero P, Pacchiana G, Capra M, Christensen J, et al: ATAD2 is a novel cofactor for MYC, overexpressed and amplified in aggressive tumors. Cancer Res 69: 8491-8498, 2009.

7. Wu G, Lu X, Wang Y, He H, Meng X, Xia S, Zhen K and Liu Y: Epigenetic high regulation of ATAD2 regulates the Hh pathway in human hepatocellular carcinoma. Int J Oncol 45: 351-361, 2014.

8. Revenko AS, Kalashnikova EV, Gemo AT, Zou JX and Chen HW: Chromatin loading of E2F-MLL complex by cancer-associated coregulator ANCCA via reading a specific histone mark. Mol Cell Biol 30: 5260-5272, 2010.

9. Hsia EY, Kalashnikova EV, Revenko AS, Zou JX, Borowsky AD and Chen HW: Deregulated E2F and the AAA+ coregulator ANCCA drive proto-oncogene ACTR/AIB1 overexpression in breast cancer. Mol Cancer Res 8: 183-193, 2010.

10. Wan WN, Zhang YX, Wang XM, Liu YJ, Zhang YQ, Que YH and Zhao WJ: ATAD2 is highly expressed in ovarian carcinomas and indicates poor prognosis. Asian Pac J Cancer Prev 15 2777-2783, 2014

11. Raeder MB, Birkeland E, Trovik J, Krakstad C, Shehata S, Schumacher S, Zack TI, Krohn A, Werner HM, Moody SE, et al: Integrated genomic analysis of the 8q24 amplification in endometrial cancers identifies ATAD2 as essential to MYC-dependent cancers. PLoS One 8: e54873, 2013.

12. Zhang Y, Sun Y, Li Y, Fang Z, Wang R, Pan Y, Hu H, Luo X, Ye T, $\mathrm{Li} \mathrm{H}$, et al: ANCCA protein expression is a novel independent poor prognostic marker in surgically resected lung adenocarcinoma. Ann Surg Oncol 20 (Suppl 3): S577-S582, 2013.

13. Wu G, Liu H, He H, Wang Y, Lu X, Yu Y, Xia S, Meng X and Liu Y: miR-372 down-regulates the oncogene ATAD2 to influence hepatocellular carcinoma proliferation and metastasis. BMC Cancer 14: 107, 2014

14. Alizadeh AA, Eisen MB, Davis RE, Ma C, Lossos IS Rosenwald A, Boldrick JC, Sabet H, Tran T, Yu X, et al: Distinct types of diffuse large B-cell lymphoma identified by gene expression profiling. Nature 403: 503-511, 2000

15. Creech GS, Paresi C, Li YM and Danishefsky SJ: Chemical synthesis of the ATAD2 bromodomain. Proc Natl Acad Sci USA 111: 2891-2896, 2014.

16. Yang P, Guo L, Duan ZJ, Tepper CG, Xue L, Chen X, Kung HJ, Gao AC, Zou JX and Chen HW: Histone methyltransferase NSD2/MMSET mediates constitutive NF- $\kappa$ B signaling for cancer cell proliferation, survival, and tumor growth via a feed-forward loop. Mol Cell Biol 32: 3121-3131, 2012.
17. Dhalluin C, Carlson JE, Zeng L, He C, Aggarwal AK and Zhou MM: Structure and ligand of a histone acetyltransferase bromodomain. Nature 399: 491-496, 1999.

18. Jacobson RH, Ladurner AG, King DS and Tjian R: Structure and function of a human TAFII250 double bromodomain module. Science 288: 1422-1425, 2000.

19. Shahbazian MD and Grunstein M: Functions of site-specific histone acetylation and deacetylation. Annu Rev Biochem 76: 75-100, 2007

20. Zou JX, Guo L, Revenko AS, Tepper CG, Gemo AT, Kung HJ and Chen HW: Androgen-induced coactivator ANCCA mediates specific androgen receptor signaling in prostate cancer. Cancer Res 69: 3339-3346, 2009.

21. Caron C, Lestrat C, Marsal S, Escoffier E, Curtet S, Virolle V, Barbry P, Debernardi A, Brambilla C, Brambilla E, et al: Functional characterization of ATAD2 as a new cancer/testis factor and a predictor of poor prognosis in breast and lung cancers. Oncogene 29: 5171-5181, 2010.

22. Kalashnikova EV, Revenko AS, Gemo AT, Andrews NP, Tepper CG, Zou JX, Cardiff RD, Borowsky AD and Chen HW: ANCCA/ATAD2 overexpression identifies breast cancer patients with poor prognosis, acting to drive proliferation and survival of triple-negative cells through control of B-Myb and EZH2. Cancer Res 70: 9402-9412, 2010.

23. Fouret R, Laffaire J, Hofman P, Beau-Faller M, Mazieres J, Validire P, Girard P, Camilleri-Bröet S, Vaylet F, Leroy-Ladurie F, et al: A comparative and integrative approach identifies ATPase family, AAA domain containing 2 as a likely driver of cell proliferation in lung adenocarcinoma. Clin Cancer Res 18: 5606-5616, 2012.

24. Hsia EY,Zou JX and Chen HW: The roles and action mechanisms of p160/SRC coactivators and the ANCCA coregulator in cancer. Prog Mol Biol Transl Sci 87: 261-298, 2009.

25. Duan Z, Zou JX, Yang P, Wang Y, Borowsky AD, Gao AC and Chen HW: Developmental and androgenic regulation of chromatin regulators EZH2 and ANCCA/ATAD2 in the prostate via MLL histone methylase complex. Prostate 73: 455-466, 2013.

26. Zhou Y, Sui C, Li B, Yin Z, Tan Y, Yang J and Liu Z: Repeat hepatectomy for recurrent hepatocellular carcinoma: a local experience and a systematic review. World J Surg Oncol 8: 55, 2010.

27. De Angelis PM, Svendsrud DH, Kravik KL and Stokke T: Cellular response to 5-fluorouracil (5-FU) in 5-FU-resistant colon cancer cell lines during treatment and recovery. Mol Cancer 5: 20, 2006.

28. Zhang L, Hao Q, Bao L, Liu W, Fu X, Chen Y and Wu H: Phenethyl issothiocyanate suppresses cervical carcinoma metastasis potential and its molecular mechanism. Mol Med Rep 10: 2675-2680, 2014. 